\title{
CUX1, a haploinsufficient tumour suppressor gene overexpressed in advanced cancers
}

\section{Zubaidah M. Ramdzan ${ }^{1}$ and Alain Nepveu ${ }^{1-4}$}

Abstract | CUT-like homeobox 1 (CUX1) is a homeobox gene that is implicated in both tumour suppression and progression. The accumulated evidence supports a model of haploinsufficiency whereby reduced CUX1 expression promotes tumour development. Paradoxically, increased CUX1 expression is associated with tumour progression, and ectopic CUX1 expression in transgenic mice increases tumour burden in several tissues. One CUX1 isoform functions as an ancillary factor in base excision repair and the other CUX1 isoforms act as transcriptional activators or repressors. Several transcriptional targets and cellular functions of CUX1 affect tumorigenesis; however, we have yet to develop a mechanistic framework to reconcile the opposite roles of CUX1 in cancer protection and progression.

Loss-of-heterozygosity (LOH). Loss of one allele of a gene when the original two alleles can be distinguished. This is common for tumour suppressor genes when the other allele is mutated, although it may occur without mutation of the remaining allele.

'Goodman Cancer Centre McGill University, 1160 Pine Avenue West, Montreal, Quebec, H3A 1A3, Canada. ${ }^{2}$ Department of Biochemistry, McGill University, 1160 Pine Avenue West, Montreal, Quebec, H3A 1A3, Canada ${ }^{3}$ Department of Medicine, McGill University, 1160 Pine Avenue West, Montreal, Quebec, H3A 1A3, Canada. ${ }^{4}$ Department of Oncology, McGill University, 1160 Pine Avenue West, Montreal, Quebec, H3A 1A3, Canada. Correspondence to A.N. e-mail: alain.nepveu@mcgill.ca doi:10.1038/nrc3805 Published online

5 September 2014

\section{This Review is dedicated to the memory of Rosalind Goodman.}

CUT-like homeobox 1 (CUX1) is the mammalian orthologue of the Drosophila melanogaster cut (ct) gene $^{1}$. The human CUX1 gene was identified following purification of the CCAAT-displacement protein (CDP), and has also been called CUT-like 1 (CUTL1) and $\mathrm{CDP} / \mathrm{CUT}^{2}$. A second gene, called CUX2, is expressed primarily in neuronal cells and has not been linked to cancer. CUX1 has been implicated in cancer both as a tumour suppressor and an oncogene. Recent genetic mapping and expression analyses pointed to CUX1 as the tumour suppressor that is the target of loss-of-heterozygosity (LOH) on chromosome 7q22.1 (REFS 3-6). In cancers with CUX1 LOH, no mutations have been found in the remaining allele ${ }^{7-10}$ and, in tested cases, CUX1 was expressed, albeit at a reduced level ${ }^{4,5,11}$. However, inactivating point mutations were shown in $1-5 \%$ of cancers in which the two CUX1 alleles are present ${ }^{11}$ (FIG. 1). The accumulated evidence is therefore consistent with the notion that CUX1 is a haploinsufficient tumour suppressor gene.

Paradoxically, increased CUX1 expression is frequently observed in various cancers and is associated with shorter disease-free survival ${ }^{12-14}$. Consistent with this, transgenic mice expressing various CUX1 isoforms exhibit multiorgan hyperplasia ${ }^{15}$ and develop myeloproliferative disease (MPD)-like myeloid leukaemias ${ }^{16}$ and tumours in the mammary gland ${ }^{17-19}$, the lung ${ }^{18}$ and the uterus ${ }^{20}$. Many cell lines with CUX1 LOH that are listed on the Sanger cancer cell line website (see Further information) harbour amplification of the remaining allele, illustrating the dual role of CUX1 in cancer (TABLE 1).

Cell-based assays have shown transcriptional roles for CUX1 in cell cycle progression ${ }^{21,22}$, DNA damage responses ${ }^{23}$, and resistance to apoptotic signals ${ }^{14}$ and other pathways (FIG. 2). In addition, one CUX1 protein has a direct role in DNA repair as an accessory factor in the base excision repair pathway ${ }^{18}$. Many transcriptional targets and cellular functions of CUX1 can explain its role either in tumour suppression or tumour progression, but to reconcile the opposite effects of CUX1 in suppressing tumour formation and promoting cancer cell survival and progression will require further studies and perhaps the elaboration of novel concepts in cancer.

Below, we describe the molecular and cellular functions that have been ascribed to the main CUX1 protein isoforms. We review the genetic and experimental evidence from human cancers for the opposite roles of CUX1 in tumour suppression and tumour progression, and we detail the phenotypes of Cuxl-knockout and transgenic mouse models. Finally, we discuss the biochemical activities of CUX1 that affect cancer and illustrate two cases of non-oncogene addictions involving CUX1.

\section{Molecular and cellular functions of CUX1}

Multiple CUX1 isoforms have been described, two of which are ubiquitously expressed ${ }^{2,22,24-27}$ (reviewed in REF. 28). The full-length protein, often referred 


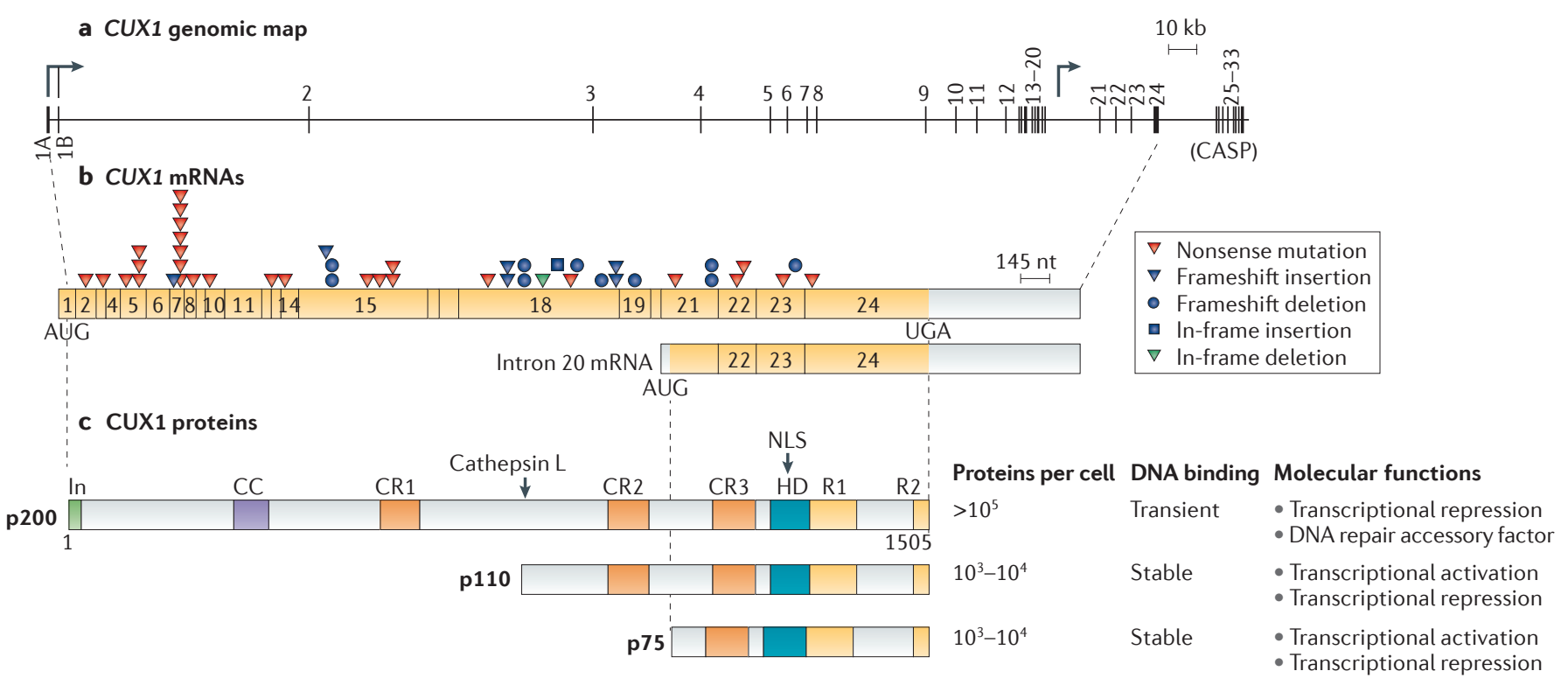

d CUX1 proteins in knockout mice Cux1 $1^{\text {lacZ }}$ LacZ

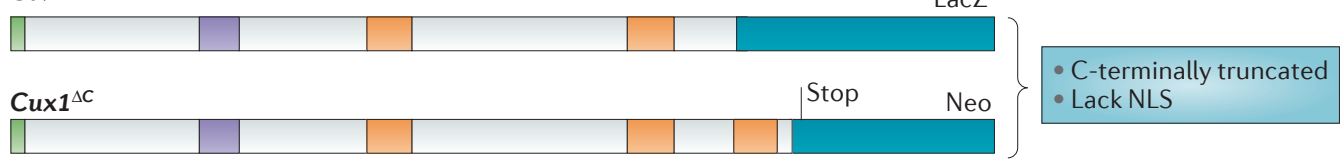

Figure 1 | Structure of the CUT-like homeobox 1 (CUX1) gene, mRNAs and proteins. a | Vertical lines represent individual exons. Transcription starts at exon $1 \mathrm{~A}$ or $1 \mathrm{~B}$, or within intron 20 , and can end after exons 24 or 33. b | The two main CUX1 mRNAs are shown. Indicated above are loss-of-function somatic mutations, as described in REF. 11. Not shown is the CDP/CUT alternatively spliced product (CASP), which is spliced between exon 14 and 25 and ends at exon 33. c | The full-length p200 CUX1 protein is proteolytically processed by cathepsin L to generate the p110 CUX1 isoform, whereas p75 CUX1 is encoded by the intron 20 mRNA. Evolutionarily conserved regions are shown: coiled-coil (CC), CUT repeat 1 (CR1), CR2 and CR3, and the CUT homeodomain (HD). An autoinhibitory (In) domain is present at the amino-terminus, and two active repression domains, R1 and R2, are located in the carboxy-terminal region. $\mathbf{d} \mid \mathrm{CUX} 1$ proteins expressed in knockout mice. One gene inactivation strategy involved replacement of the CR3 exon with $\beta$-galactosidase coding sequences, to make a CUX1-LacZ fusion protein ${ }^{35}$. In the other strategy, a neomycin resistance (Neo) gene cassette with an in-frame termination codon was inserted in place of the CUT HD exon ${ }^{50,51}$. kb, kilobase; NLS, nuclear localization signal; nt, nucleotide. Part b is modified with permission from REF. 11, Nature Publishing Group.

Haploinsufficient Describing loss or mutagenic inactivation of a single allele of a tumour suppressor gene that hastens tumorigenicity.

Non-oncogene addictions The concept of 'non-oncogene addiction' describes the heightened dependency of tumour cells on the normal cellular functions of certain genes that are not themselves classical oncogenes. to as p200 CUX1, contains four evolutionarily conserved DNA-binding domains: that is, three CUT repeats (CR1, CR2 and CR3) and a CUT homeodomain (HD) ${ }^{2}$. On the basis of reporter assays and in vitro DNA binding assays, early studies described p200 CUX1 as a transcriptional repressor that functions in myeloid precursor cells to downregulate the expression of genes that become expressed only in terminally differentiated cells $\mathrm{s}^{29-33}$. However, it has not been possible to confirm the recruitment of p200 CUX1 to specific genomic sites in vivo using chromatin immunoprecipitation, because it is very difficult to immunoprecipitate the full-length CUX1 protein following cross-linking (R. Harada and A.N., unpublished observation). Moreover, immunohistochemical evidence indicates that CUX1 is expressed in terminally differentiated cells of several tissues, including neurons of the cerebral cortex ${ }^{14,34,35}$. p200 CUX1 is abundant and binds DNA rapidly but only transiently ${ }^{36}$. These properties are not consistent with a role as a classical transcription factor that stably binds to DNA and recruits a co-activator or a co-repressor, but it is still possible for this protein to repress transcription by competition for occupancy of the binding site $^{37}$. Indeed, as mentioned above, CUX1 was originally purified as $\mathrm{CDP}^{2,29}$. In addition to its presumed role in transcriptional repression, it was recently shown that p200 CUX1 has a direct role in DNA repair through its three CUT repeat domains ${ }^{18}$. CUT repeats function as accessory factors in base excision repair (BER) - the pathway that repairs most oxidative DNA damage lesions, including oxidized bases, apurinic and apyrimidinic sites and single-strand breaks ${ }^{38}$. Single-cell gel electrophoresis (also known as the comet assay) showed that CUX1 knockdown or genetic inactivation of CUX1 impairs DNA repair following treatment with ionizing radiation or hydrogen peroxide ${ }^{39}$. By contrast, ectopic CUX1 expression accelerates DNA repair ${ }^{18}$. In vitro DNA repair assays established that recombinant proteins with various combinations of CUT repeat domains can stimulate the enzymatic activities of 8-oxoguanine DNA glycosylase (OGG1) - a major enzyme in base excision repair ${ }^{18}$.

In mid-G1 phase, $1 \%$ to $5 \%$ of p 200 CUX1 is proteolytically processed to generate p110 CUX1, which contains the last two CUT repeats and the CUT HD (CR2-CR3-HD) $)^{25,40}$. This isoform, although produced 
Table 1 | CUX1 copy number variations in human tumours and cancer cell lines*

\begin{tabular}{|llll}
\hline Type & $\begin{array}{l}\text { Number of tumours (percentage } \\
\text { of all tumours studied) }\end{array}$ & $\begin{array}{l}\text { Number of cell lines (percentage } \\
\text { of all cell lines studied) }\end{array}$ & $\begin{array}{l}\text { Copy } \\
\text { number }\end{array}$ \\
\hline Amplification $^{\ddagger}$ & $36(3 \%)$ & $5(1 \%)$ & $\geq 8$ \\
\hline Gain $^{\S}$ & $885(71 \%)$ & $311(71 \%)$ & $3-7$ \\
\hline Loss $^{\|}$ & $10(9 \%)$ & $45(10 \%)$ & $2-4$ \\
\hline LOH and gain & $29(2 \%)$ & $25(6 \%)$ & $3-10$ \\
\hline LOH & $187(15 \%)$ & $52(12 \%)$ & $1-3$
\end{tabular}

CUX1, CUT-like homeobox 1; LOH, loss-of-heterozygosity. *Data taken from the Catalogue of Somatic Mutations in Cancer (COSMIC) (see Further information), 25 April 2014. ${ }^{\ddagger}$ On the COSMIC website, amplification is defined as total copy number $>8 .{ }^{8} \mathrm{On}$ the COSMIC website, gain is defined as total copy number $\geq$ average genome ploidy +0.6 . "On the COSMIC website, loss is defined as total copy number $\leq$ average genome ploidy -0.6 .

at low levels, stably interacts with DNA and functions as a transcriptional repressor or activator depending on promoter context $\mathrm{t}^{25,39,41}$. Using transcription and cellbased assays, a role for p110 CUX1 was shown in many cellular processes, notably in cell cycle progression and cell proliferation ${ }^{21,22}$, strengthening of the spindle assembly checkpoint ${ }^{19}$, establishment of a transcriptional programme that enables efficient DNA damage response ${ }^{23}$, and cell migration and invasion ${ }^{13,42}$. In addition, from RNA interference (RNAi)-mediated knockdown and genetic inactivation, CUX1 was shown to be required for the resistance to apoptotic signals in pancreatic cancer cells $^{14}$, the repression cytokine genes associated with M1 macrophages ${ }^{43}$, and dendrite branching and spine development in cortical neurons ${ }^{34}$. Which isoform is responsible for these functions remains to be established. Note also that although the p110 CUX1 isoform contains two CUT repeats and therefore has the potential to participate in DNA repair transactions, it is unlikely to have a substantial effect in this process, as it is present at only a few thousand copies per cell, at the most.

\section{CUX1 as a tumour suppressor gene}

LOH of 7q22. CUX1 is located at chromosome 7q22.1, a region that sustains frequent $\mathrm{LOH}$ in several cancers, notably in $14 \%$ of uterine leiomyomas ${ }^{44,45}, 18 \%$ of breast cancers $^{46}, 15-25 \%$ of acute myeloid leukaemias (AMLs) and $\mathrm{MPDs}^{47}$, and in up to $40 \%$ of therapy-associated MPD and $\mathrm{AML}^{48}$. Although early cytogenetic studies and polymorphic marker analyses clearly pointed to the presence of a tumour suppressor gene on 7q22.1, the implicated gene was not rapidly identified. CUX1 and a few other genes were consistently present within the smallest deleted region, but no mutation was found in the remaining allele of any of these genes ${ }^{7-10}$. These results eventually led to the idea that inactivation of the tumour suppressor on 7q22.1 may not conform to the Knudson two-hit model.

Monoallelic loss of CUX1. Several recent studies have pointed to CUX1 as the putative haploinsufficient tumour suppressor gene on $7 q 22.1$. In uterine leiomyomas, a positional cloning approach revealed two cases of genomic rearrangements with breakpoints predicted to inactivate one CUX1 allele ${ }^{3}$. High-resolution single-nucleotide polymorphism (SNP) microarray analysis indicated that progression of Philadelphia chromosome-negative myeloproliferative neoplasms (MPNs) to AML was associated with frequent $\mathrm{LOH}$ of the $7 q 22.1$ chromosomal region ${ }^{6,10}$. In one study, mapping of the commonly deleted region identified CUX1 as the single target gene $e^{6}$. In the second study, the minimally deleted region included only two target genes, CUX1 and SH2B2 (REF. 10). A follow-up study of $15 \mathrm{sec}-$ ondary AML cases by the same group detected a hemizygous missense substitution (V1288L) in the homeobox of CUX1 (REF. 49). Two additional studies, using SNP array analysis on de novo and therapy-related myeloid neoplasms, identified CUX1 in the commonly deleted region of 7q22.1 (REFS 4,5). RNA sequencing and reverse transcription PCR analysis showed that CUX1 mRNA expression was reduced approximately twofold in leukaemic cells of affected patients $s^{4,5}$, and immunoblotting using a carboxy-terminal antibody showed that the fulllength CUX1 protein was reduced in AML cell lines with chromosome 7 and chromosome 7q loss karyotypes ${ }^{5}$. Reduced CUX1 mRNA expression was also documented in an AML sample that had a chimeric transcript containing CUX1 exon 1 upstream of claudin 7 (CLDN7) exons $2-4$, probably resulting from a chromosomal translocation ${ }^{5}$.

Inactivating point mutations in one allele. Although no mutations were found in the remaining allele in cancers with $C U X 1 \mathrm{LOH}^{7-10}$, inactivating point mutations were found in $1-5 \%$ of cancers in which the two alleles are present ${ }^{11}$. The Adams group from the Wellcome Trust Sanger Institute, Hinxton, Cambridgeshire, UK, analysed 7,651 human cancer genomes of various tissue types to identify cancer driver genes that had undergone loss-of-function mutations. CUX1 was one of 54 genes showing a higher ratio of observed to expected nonsense mutations ${ }^{11}$. Point mutations in CUX1 were found in $0.7 \%$ to $5 \%$ of tumours, depending on the tissue of origin, and included approximately $21 \%$ of nonsense and frameshift mutations. The effect of these mutations is to generate a C-terminally truncated protein that, most of the time, lacks the nuclear localization signal located in the CUT HD (FIG. 1). As the protein remains in the cytoplasm, these mutations effectively inactivate the function of CUX1, as shown by 


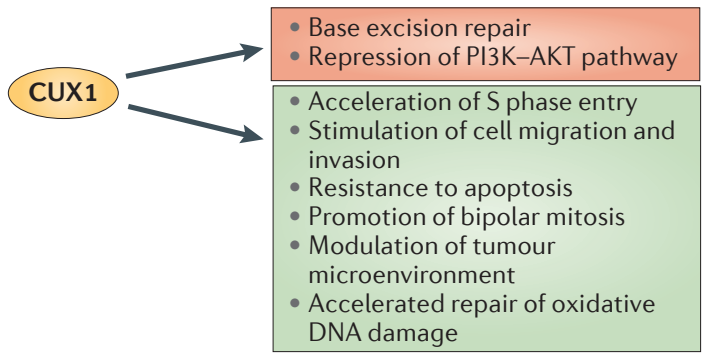

Figure 2 | Mechanisms of action in cancer. Cellular functions of CUT-like homeobox 1 (CUX1) that are consistent with a role as a tumour suppressor (shown in the red box) include the direct stimulation of 8-oxoguanine DNA glycosylase (OGG1; a DNA glycosylase that is involved in base excision repair) and the transcriptional activation of phosphoinositide-3-kinase interacting protein 1 (PIK3IP1; an inhibitor of the PI3K-AKT signalling pathway). Cellular functions that can promote tumour progression (shown in the green box) include acceleration of cell cycle progression and cell proliferation, stimulation of cell migration and invasion, increased resistance to apoptosis, reinforcement of the spindle assembly checkpoint to promote bipolar mitosis, modulation of the tumour microenvironment and acceleration of oxidative DNA damage repair.

the multiple phenotypes of two Cux1-knockout mouse models (discussed below) that were generated through a similar strategy ${ }^{35,50,51}$. In addition, approximately $40 \%$ of missense mutations were predicted to be deleterious by two independent algorithms $\mathrm{s}^{52,53}$. Inactivating mutations were most frequent in cancers of the endometrium, large intestine and lung. Although LOH of CUX1 is most frequent in AML and MPDs, screening of 738 patients with myelodysplasia and MPNs identified inactivating mutations in only $2 \%$ of cases.

Commonly deleted regions on $7 \mathrm{q}$ in myeloid disorders include not only $7 \mathrm{q} 22$, but also $7 \mathrm{q} 34$ and 7q35-7q36.1 (REF. 4). The complexity of 7q rearrangements suggests that multiple genetic factors, rather than a single tumour suppressor gene, contribute to the pathogenesis of myeloid disorders. Indeed, CUX1 inactivating mutations are associated with poorer overall survival in a cohort of patients with myelodysplasia or MPN, and in a cohort with AML, but the overall outcome was significantly worse among patients with loss of chromosome 7 or deletion of chromosome $7 \mathrm{q}(\operatorname{del}(7 \mathrm{q}))^{11}$.

Although genetic evidence indicates that one CUX1 allele remains intact in all cases of $\mathrm{LOH}$ or inactivating point mutations, two patients with post-MPN AML harboured a homozygous deletion spanning CUX1 (REFS 6,10), and another patient with chronic myelomonocytic leukaemia had heterozygous nonsense CUX1 mutations ${ }^{11}$. It is therefore possible that in rare cases, CUX1 is inactivated like a classical tumour suppressor gene.

In vivo evidence that CUX1 is a tumour suppressor gene. In D. melanogaster, RNAi-mediated knockdown of ct in developing haemocytes led to the development of melanotic pseudotumours ${ }^{5,11}$, and $c t$ knockdown in the proliferating eye disc increased the overproliferation phenotype caused by overexpression of the Notch-ligand Delta $^{11}$. In human cord blood progenitors, partial knockdown of CUX1 led to a $40 \%$ increase in engraftment on transplantation into immunodeficient mice ${ }^{5}$. CUX1 knockdown in KE37 T cell acute lymphoblastic leukaemia (T-ALL) cells increased tumour formation following subcutaneous injection into immunodeficient mice ${ }^{11}$. Another approach exploited a transposon-mediated mutagenesis screen in mouse haematopoietic tissues ${ }^{54}$. Sense and antisense insertions of the Sleeping Beauty T2/Onc transposon in Cuxl were documented in 45\% (20/44) of T-ALLs that developed after activation of the transposon and were associated with a $\sim 50 \%$ reduction in levels of Cux1 mRNA and p200 CUX1 protein ${ }^{11}$. These results clearly establish that reduced CUX1 expression can promote proliferation; however, it should be noted that the affected cells are of different types than the human cancers in which CUX1 LOH or loss of function mutations are found.

Knockout mice provide limited evidence. Two Cux 1 mouse knockouts have been generated and have been analysed by several groups ${ }^{35,50,51}$. In both cases, the gene inactivation strategy led to the production of a protein that is truncated upstream of the CUT HD and is therefore not imported into the nucleus ${ }^{35,50}$. Cux1 heterozygous mice were indistinguishable from wild-type mice and were not further investigated. The effect of Cuxl hemizygosity on tumour incidence therefore remains to be investigated. In $\mathrm{Cuxl}^{-/-}$homozygous mice, severe phenotypes were documented and only a few mice survived to weaning age, preventing an assessment of the effect of Cuxl inactivation on tumour incidence (reviewed in REF. 28). However, some observations are relevant to the role of CUX1 in cancer. Mouse embryonic fibroblasts (MEFs) that were derived from $\mathrm{Cux1^{-/ }}$ mice showed a longer G1 phase and proliferated more slowly than their wild-type counterparts ${ }^{21}$. Myeloid hyperplasia was observed in bone marrow, the spleen and the peripheral blood of $\mathrm{Cux1^{-1- }}$ mice, an observation that fits well with frequent $C U X 1 \mathrm{LOH}$ reported in $\mathrm{MPDs}^{51}$. By contrast, increased apoptosis was found to cause a twofold to threefold reduction in the percentage and absolute numbers of B cells and a fivefold reduction in thymocytes in the thymus. Bone marrow reconstitution experiments indicated that both cell-intrinsic and microenvironmental effects were implicated in the demise of lymphoid cells, leading the authors to propose that CUX1 might upregulate survival factors or downregulate death-inducing factors ${ }^{51}$. Confirmation of these two hypotheses was later provided in an independent study showing that RNAi-mediated knockdown of CUX1 leads to upregulation and downregulation of tumour necrosis factor- $\alpha$ (TNF $\alpha)$ and BCL-2, respectively ${ }^{14}$. Indeed, TNFa expression was increased in several tissues of $\mathrm{Cux1}^{-1-}$ mice ${ }^{51}$, and many phenotypes of

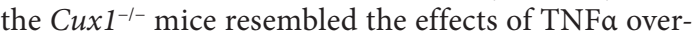
expression, including alopecia, cachexia, lymphopenia and myeloid hyperplasia ${ }^{51,55}$. In summary, Cuxl gene inactivation in the mouse caused an increase in myeloid cells but a decrease in other cell types. 


\section{Evidence that CUX1 is an oncogene}

Genetic data from human cancers. Paradoxically, copy number variation $(\mathrm{CNV})$ analysis indicates that gains are much more frequent than losses in cancer of many tissues, including cancers that harbour a high frequency of loss-of-function mutations (TABLES 1,2). For example, point mutations in CUX1 are relatively frequent (5.29\%) in cancers of the large intestine, but copy number reduction and gain are observed in $2.9 \%$ and $37.2 \%$ of these cancers, respectively (TABLE 2). Frequent copy number gain is in agreement with results from the comprehensive molecular characterization of human colon and rectal cancer in which CUX1 was ranked as the fifth gene on a scale showing a correlation between tumour aggressiveness and a combined score based on gene expression and somatic CNVs ${ }^{12}$. An increase in CUX1 copy number is also observed in cancers of the central nervous system (70.6\%), endometrium (12.2\%), kidney (29\%), lung (35.1\%), ovary (33.5\%), pancreas $(6.9 \%)$ and parathyroid (6.9\%) (TABLE 2). The only cancers in which copy number loss is more frequent than gain are those of haematopoietic and lymphoid tissues (8.3\% loss versus $0.5 \%$ gain). Overall, findings from $\mathrm{LOH}$ and point mutation analyses indicate partial loss-of-function of CUX1 in some cancers, whereas CNV data suggest increased CUX1 activity. When loss and increased function are observed in cancers of the same tissue-of-origin, it is not clear whether these genetic events each define distinct types of cancers or whether both occur successively in the same tumour. Analysis of cancer cell lines may be informative in this regard. Interestingly, approximately one-third (25 of 77) of cell lines with CUX1 LOH show amplification of the remaining allele (TABLE 1). To explain these findings, the most plausible sequence of events is that one allele is inactivated first, and the remaining allele is amplified later (FIG. 3). Such a scenario is compatible with the notion that decreased CUX1 expression facilitates tumour initiation, whereas increased CUX1 expression promotes tumorigenic progression.

CUX1 expression in human cancers. Data on CUX1 expression in human cancers are relatively limited. One problem resides in the complex structure of the gene, which contains 25 exons, and the fact that, until recently, expression profiling studies used microarrays (FIG. 1). As microarray probes are often chosen from the mRNA 3' untranslated region, most, and in some cases all, probes for CUX1 on commercially available microarrays are specific for the CDP/CUT alternatively spliced product (CASP), a Golgi resident protein ${ }^{56,57}$. Consequently, expression profiles based on microarray data do not provide useful information on CUX1 expression. A second problem stems from the fact that antibodies that recognize the $110 \mathrm{CUX} 1$ processed isoform also recognize the p200 CUX1 full-length protein, which is more than 20 times more abundant. Immunohistochemical analysis therefore provides information only on p200 CUX1.

In situ hybridization on multiple tissue core arrays showed increased CUX1 expression within high-grade, but not low-grade, breast carcinomas ${ }^{13}$. Moreover, among patients with grade 3 breast tumours, CUX1

\begin{tabular}{|c|c|c|c|c|}
\hline \multirow[t]{2}{*}{ Cancer type } & \multicolumn{2}{|l|}{ Point mutations } & \multicolumn{2}{|c|}{ Copy number variation } \\
\hline & $\begin{array}{l}\text { Percentage } \\
\text { mutated (number) }\end{array}$ & $\begin{array}{l}\text { Number of } \\
\text { samples analysed }\end{array}$ & $\begin{array}{l}\text { Variant percentage } \\
\text { (number) }\end{array}$ & $\begin{array}{l}\text { Number of } \\
\text { samples analysed }\end{array}$ \\
\hline Breast & $0.69 \%(7)$ & 1,015 & $\begin{array}{l}\text { - Gain, 15.6\% (133) } \\
\text { - Loss, 15.7\% (134) }\end{array}$ & 852 \\
\hline $\begin{array}{l}\text { Central nervous } \\
\text { system }\end{array}$ & $0.17 \%(1)$ & 573 & $\begin{array}{l}\text { - Gain, 70.6\% (290) } \\
\text { - Loss, } 1.9 \%(8)\end{array}$ & 411 \\
\hline Cervix & $14.3 \%(2)$ & 14 & NA & 0 \\
\hline Endometrium & $8.5 \%(24)$ & 281 & $\begin{array}{l}\text { - Gain, } 12.2 \%(30) \\
\text { - Loss, } 8.9 \%(22)\end{array}$ & 246 \\
\hline $\begin{array}{l}\text { Haematopoietic } \\
\text { and lymphoid }\end{array}$ & $0.28 \%(3)$ & 1,057 & $\begin{array}{l}\text { - Gain, 0.5\% (1) } \\
\text { - Loss, } 8.3 \%(16)\end{array}$ & 192 \\
\hline Kidney & $1.26 \%(6)$ & 475 & $\begin{array}{l}\text { - Gain, 29\% (87) } \\
\text { - Loss, 2.9\% (9) }\end{array}$ & 300 \\
\hline Large intestine & $5.29 \%(33)$ & 636 & $\begin{array}{l}\text { - Gain, 37.2\% (181) } \\
\text { - Loss, } 2.9 \% \text { (14) }\end{array}$ & 486 \\
\hline Liver & $1.18 \%(5)$ & 424 & NA & 0 \\
\hline Lung & $4.07 \%(35)$ & 861 & $\begin{array}{l}\text { - Gain, 35.1\% (167) } \\
\text { - Loss, 11.8\% (56) }\end{array}$ & 476 \\
\hline Not specified & $0.43 \%(1)$ & 235 & Loss $10.0 \%$ (3) & 30 \\
\hline Ovary & $1.79 \%(9)$ & 504 & $\begin{array}{l}\text { - Gain 33.5\% (155) } \\
\text { - Loss } 11.7 \% \text { (54) }\end{array}$ & 462 \\
\hline Pancreas & $0.26 \%(1)$ & 388 & Gain $6.9 \%(2)$ & 29 \\
\hline
\end{tabular}

CUX1, CUT-like homeobox 1; NA, not analysed. *Data taken from the Catalogue of Somatic Mutations in Cancer (COSMIC) (see Further information), 25 April 2014. 


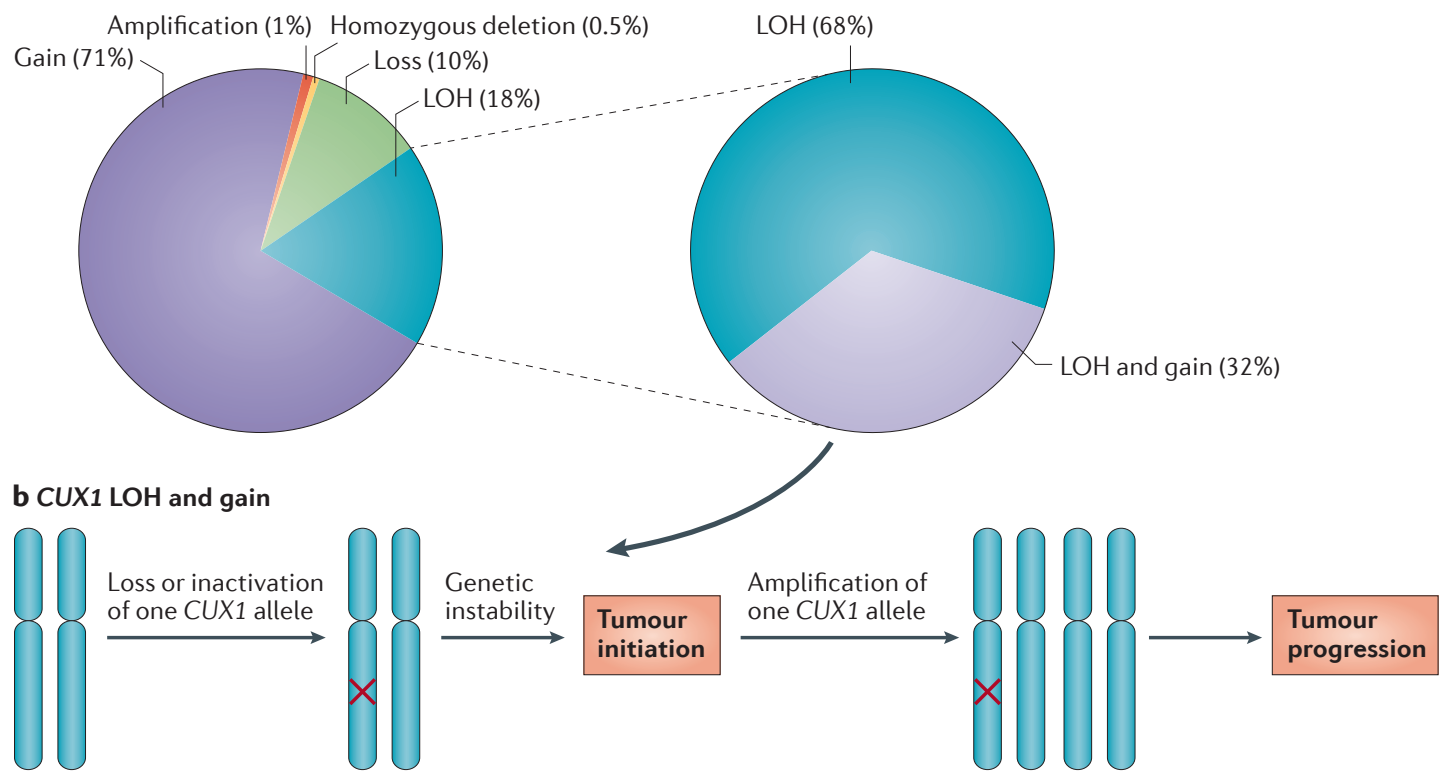

Figure 3 | CUT-like homeobox 1 (CUX1) copy number variations in human tumours and cancer cell lines. a | 25 of 77 (32\%) cell lines with CUX1 loss-of-heterozygosity $(\mathrm{LOH})$ display amplification of the remaining allele (TABLE 1). b | Deletion of one allele in cancer cells with CUX1 amplification would not confer a new phenotype. Therefore, the reverse order of events must occur during tumour development: one allele is inactivated first, either as the result of LOH or inactivating somatic point mutations, and the remaining allele is amplified later. Such a scenario suggests that CUX1 expression facilitates tumour initiation, whereas increased CUX1 expression is selected later during tumour progression. This hypothetical model remains to be rigorously tested.

mRNA expression inversely correlated with relapsefree and overall survival ${ }^{13}$. Immunohistochemical analysis on separate series of breast and pancreatic cancers confirmed that p200 CUX1 protein expression was increased in the high histological grade tumours compared with low-grade tumours ${ }^{13,58}$. Interestingly, CUX1 mRNA and protein expression is increased by TGF $\beta$ and is required for TGF $\beta$-induced cell migration and inva$\operatorname{sion}^{13,43}$. An alternative CUX1 transcript that is initiated within intron 20 and codes for the p75 isoform (FIG. 1) is expressed specifically in the testis and thymus ${ }^{24,26}$. This transcript was found to be aberrantly expressed in many breast tumour cells lines and breast tumours, in which a significant association was established with a diffuse infiltrative growth pattern ${ }^{24}$. Indeed, transgenic mice expressing this transcript in mammary epithelial cells were shown to develop mammary tumours with metastasis to the lung ${ }^{17}$.

Evidence from transgenic mice. Transgenic mice expressing the full-length p200 CUX1 protein under the control of a cytomegalovirus promoter had striking multi-organ hyperplasia and organomegaly ${ }^{15}$. Further characterization of these mice revealed glomerulosclerosis and interstitial fibrosis in the kidney ${ }^{59}$, and hepatomegaly was associated with inflammation and biliary cell hyperplasia ${ }^{60}$. Expression of the full-length p200 CUX1 protein under the control of the mouse mammary tumour virus long terminal repeat (MMTV-LTR) led to the development of mammary tumours of diverse histopathological types with a long latency and a penetrance of $21 \%$ (REF. 18).
In addition, primary lung tumours were observed in $20 \%$ of transgenic mice ${ }^{18}$. Interestingly, $45 \%$ of mammary tumours from MMTV-p200 CUX1 transgenic mice harboured a spontaneous activating mutation (G12V or Q61L) within Kras. Cooperation between KRAS-G12V and CUX1 was confirmed using lentiviral infection in the lung. Functional analysis showed that p200 CUX1 is directly involved in DNA repair and prevents RAS-induced senescence by accelerating the repair of oxidative DNA damage ${ }^{18}$.

Transgenic mice expressing the p75 CUX1 or p110 CUX1 isoform under the control of the MMTV-LTR regulatory sequences and integrated into the hypoxanthine guanine phosphoribosyl transferase (Hprt) locus also developed mammary tumours after a long latency period, and metastasis to the lung was observed in a small proportion of p75 CUX1 transgenic mice ${ }^{17}$. However, activating mutations in Kras were found in less than 10\% of these tumours, and no mutation was found in Hras or Nras (Z.M.R. and A.N., unpublished observations). Strikingly, all tumours contained a majority of cells with a sub-tetraploid chromosome content, suggesting that they derived from a tetraploid intermediate ${ }^{19}$. p110 CUX1 was shown to upregulate many genes involved in the spindle assembly checkpoint, thereby delaying cell division and enabling bipolar mitosis in the presence of multiple centrosomes ${ }^{19}$. In addition to mammary tumours, a number of sarcomas with features resembling those of histiocytic sarcomas were observed in the uterus and liver of MMTV-p110 CUX1 and MMTV-p75 CUX1 transgenic mice ${ }^{20}$. Although mammary tumours 
and sarcomas were observed in mice of the FVB genetic background, expression of MMTV-p75 CUX1 in mice of mixed genetic backgrounds (129/Ola x FVB or 129/Ola x C57BL/6) caused a disease defined as an MPD-like myeloid leukaemia and characterized by massive expansion of neutrophils in the blood, spleen, bone marrow and non-haematopoietic organs, such as the kidneys and the lungs ${ }^{16}$. In addition, expression of the p75 CUX1 isoform under the control of the cytomegalovirus immediate early enhancer and the chicken $\beta$-actin promoter caused polycystic kidneys at variable penetrance and severity, correlating with transgene expression levels ${ }^{61}$.

In summary, expression of p200, p110 and p75 CUX1 isoforms in transgenic mice increased tumour incidence in several organs and tissues depending on the transgene promoter and mouse genetic background.

\section{Mechanisms of action in cancer}

Functions of CUX1 that promote tumorigenicity. Many transcriptional targets and cellular functions of CUX1 readily suggest mechanisms by which increased p110 or p75 CUX1 expression might promote tumour development and progression, including acceleration of $S$ phase entry ${ }^{21,22,41,62,63}$, stimulation of cell migration and inva$\operatorname{sion}^{13,42,64-66}$, resistance to apoptosis ${ }^{14}$, and promotion of bipolar mitosis in the presence of supernumerary centrosomes ${ }^{19}$ (reviewed in REF. 67). In addition, a role in the tumour microenvironment has recently been described. TGF $\beta$ that was secreted by pancreatic ductal adenocarcinoma (PDAC) cells upregulated CUX1 expression in tumour-associated macrophages. In these cells, CUX1 repressed the expression of cytokines that were associated with M1 polarization ${ }^{43}$. The mechanism of repression was shown to involve a direct interaction between p200 CUX1 and nuclear factor- $\kappa \mathrm{B}(\mathrm{NF}-\kappa \mathrm{B})$, leading to the deacetylation of NF- $\kappa \mathrm{B}$ and inhibition of its DNA binding activity.

Functions of CUX1 that suppress tumour development. Three modes of action have recently been proposed to explain the role of CUX1 as a tumour suppressor. In one study, the authors stated that the expression of nine of ten cell cycle genes was inversely correlated with CUX1 expression levels, suggesting that CUX1 inhibits cell cycle progression $^{5}$. It is not possible to evaluate the experimental evidence, as the gene list was not provided. Certainly, the idea that CUX1 represses genes that are involved in cell cycle progression runs contrary to the bulk of the evidence showing that CUX1 stimulates expression of histone genes and many genes involved in DNA replication, while repressing expression of the cyclin-dependent kinase inhibitors p21 and p27 (REFS 15,41,60,62,68-75). Moreover, in cell-based assays, MEFs from Cux1-knockout mice showed a longer G1 phase and proliferated more slowly than their wild-type counterparts; whereas, in many cell types, ectopic expression of p110 CUX1 accelerated $S$ phase entry and stimulated proliferation ${ }^{21,22}$.

In another study, p110 CUX1 was shown to activate transcription of phosphoinositide-3-kinase interacting protein 1 (PIK3IP1), a direct inhibitor of the PI3K p110 catalytic subunit ${ }^{11,76}$. CUX1 knockdown caused a decrease in the expression of PIK3IP1 and a concomitant increase in PI3K signalling and AKT phosphorylation ${ }^{11}$. Interestingly, CUX1-deficient cell lines exhibited increased sensitivity to the pan-AKT inhibitor (MK2206) and a dual PI3K and mTOR inhibitor (NVP-BEZ235). In a separate study, activation of the PI3K-AKT signalling pathway by insulin-like growth factor 1 (IGF1) or by AKT2 overexpression led to the upregulation of CUX1, at both the mRNA and the protein level, and was associated with resistance to apoptosis, whereas treatment of cells with the PI3K inhibitor LY294002 decreased CUX1 expression and increased apoptosis ${ }^{14}$. Results from these two studies seem to be contradictory, although it is possible to envisage the existence of a feedback regulatory loop whereby PI3K-AKT stimulates the expression of CUX1, which in turn would downregulate the PI3K-AKT pathway to close the loop (FIG. 4a). This remains to be verified.

Another mechanism for the role of CUX1 as a tumour suppressor was inferred from the direct role of p200 CUX1 in base excision repair ${ }^{18}$. Indeed, Cux $1^{-/-}$ MEFs exhibit increased genomic instability ${ }^{23}$. Moreover, $\mathrm{Cu} 1^{+/-}$heterozygous MEFs are haploinsufficient for DNA repair ${ }^{18}$. Whether CUX1 hemizygosity will increase tumour incidence by increasing the frequency of mutations and/or genomic rearrangements remains to be formally tested (FIG. 4b).

\section{Non-oncogene addictions involving CUX1}

As p110 CUX1 activates distinct sets of genes involved in DNA replication ${ }^{41}$, the DNA damage response ${ }^{23}$ and the spindle assembly checkpoint ${ }^{19}$, we understand that its roles in the cell cycle are to prepare cells for DNA replication, promote maintenance of genome integrity and ensure proper chromosomal segregation at the end of the cell cycle. In addition, p200 CUX1 promotes genome stability through its role in base excision repair ${ }^{18}$. Such functions would not predict a role as an oncogene, but overexpression of either p200 or p110 CUX1 contributes to tumorigenicity in cell culture models ${ }^{21,24}$ and in transgenic mice ${ }^{16,17,19,20,61}$ (reviewed in REFS 28,67). Two studies showed that cancer cells are acutely dependent on the multiple roles of CUX1 in maintaining genome integrity ${ }^{18,19}$. Such an increased requirement for the function of an otherwise normal protein has previously been referred to as 'non-oncogene addiction' (REF. 77).

Normal cells do not thrive when tetraploid, because the presence of multiple centrosomes induces the formation of a multipolar mitotic spindle ${ }^{78-80}$. Multipolar divisions lead to catastrophic chromosome missegregation, and the progeny of such divisions are almost invariably non-viable ${ }^{79}$. Increased p110 CUX1 expression, however, activates a transcriptional programme that reinforces the spindle assembly checkpoint and delays mitosis until centrosomes have clustered to enable bipolar mitosis ${ }^{19}$. However, the passage through a multipolar intermediate before centrosome clustering enriches for merotelic chromosome attachments, leading to chromosome missegregation and the rapid generation of aneuploid populations from which tumorigenic cells emerge $\mathrm{e}^{19,78,79}$. Strikingly, mammary tumours that 
a

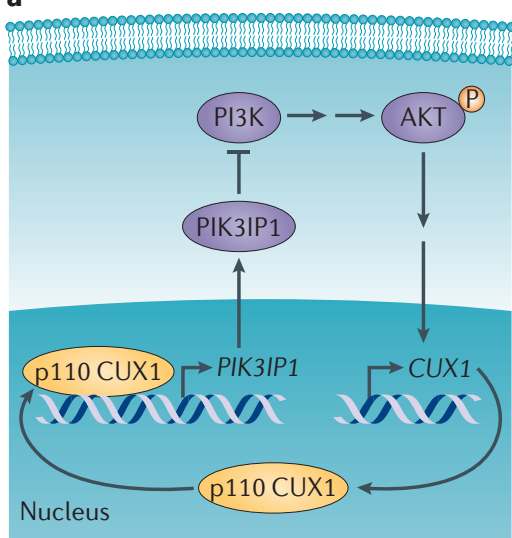

b

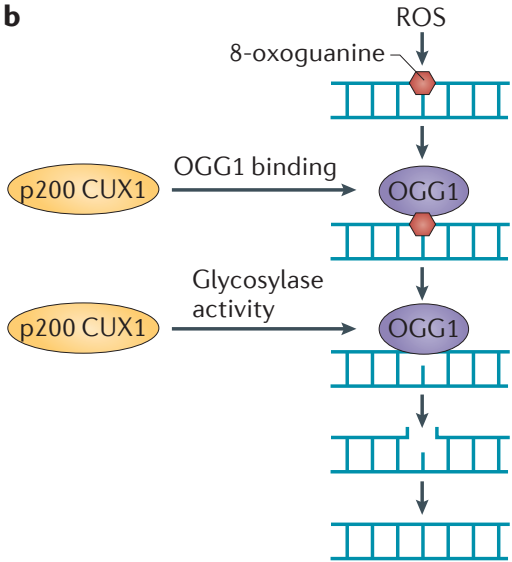

Figure 4 | Biochemical activities implicated in tumour suppression. a | The PI3K-AKT signalling pathway was shown to stimulate expression of CUT-like homeobox 1 (CUX1) ${ }^{14}$, which in turn was found to activate the phosphoinositide-3-kinase interacting protein 1 (PIK3IP1) gene ${ }^{11}$. Inactivation of one CUX1 allele was proposed to cause an increase in PI3K signalling and AKT phosphorylation $(\mathrm{P})^{11} \cdot \mathbf{b}$ | The p200 CUX1 isoform was shown to stimulate 8-oxoguanine DNA glycosylase (OGG1) DNA binding and enzymatic activity ${ }^{18}$. Increased CUX1 expression was shown to accelerate the repair of oxidative DNA damage ${ }^{18}$, and inactivation of one CUX1 allele was found to reduce DNA repair efficiency, leading to the suggestion that increased genetic instability in such cells may promote tumour initiation. ROS, reactive oxygen species.

Synthetic lethal

A situation in which the inactivation of a pathway by a genetic means is lethal to cells that harbour a mutation in an different pathway but is not overly detrimental to normal cells. arise in MMTV-CUX1 transgenic mice have a high level of aneuploidy, with most cells containing a subtetraploid chromosome content, suggesting that tumour cells in these animals arose through a process involving cytokinesis failure followed by chromosome missegregation $^{19}$. Tetraploidy is not thought to be prevalent in cancers $^{81}$; however, the importance of this mechanism in producing genetic variants in cancer was shown in a recent study of primary renal carcinomas and associated metastases ${ }^{82}$. Ploidy profiling showed that only one of eight regions of the primary tumour was tetraploid, whereas a chest-wall metastasis harboured two subtetraploid populations. Philogenetic reconstruction, based on exome sequencing and chromosome aberration analysis, showed that the metastasis evolved from the primary tumour region that was tetraploid. Two conclusions can be drawn from these findings. First, depending on which region of the primary tumour was analysed, this tumour would be classified as diploid seven times out of eight. Second, the sub-tetraploid metastatic cells originated from a tetraploid intermediate in the primary tumour.

The role of p200 CUX1 in the repair of oxidative DNA damage is essential in RAS-transformed cells. Increased levels of reactive oxygen species (ROS) in cells with sustained RAS pathway activation can cause cellular senescence; however, CUX1 prevents RASinduced senescence in primary cells. Moreover, CUX1 knockdown is synthetic lethal with oncogenic RAS in human cancer cells ${ }^{18,83}$. Strikingly, increased p200 CUX1 expression in a transgenic mouse model enables the emergence of mammary tumours with spontaneous activating Kras mutations ${ }^{18}$. Cancer cells can overcome the antiproliferative effects of excessive DNA damage by inactivating a DNA damage response pathway, such as those regulated by ataxia telangiectasia mutated (ATM) kinase or p53 signalling. These findings reveal an alternative mechanism to allow sustained proliferation in RAS-transformed cells through increased DNA base excision repair capability.

\section{Concluding remarks}

Genetic and functional evidence established that reduced levels of CUX1 promote tumour development, whereas increases in CUX1 copy number and expression are associated with tumour progression. Transgenic mouse models have established that higher expression of several CUX1 isoforms increases cancer incidence ${ }^{16,17,61}$ and that cytokinesis failure cooperates with the p75 and p110 CUX1 transcription factors in tumorigenicity ${ }^{19}$, whereas a Kras oncogene cooperates with the p200 CUX1 DNA repair accessory factor ${ }^{18}$. A hemizygous mouse knockout model should be used in the future to verify that inactivation of one Cuxl allele promotes tumorigenicity, particularly in the myeloid compartment, and to identify molecular events that cooperate with Cux1 hemizygosity in tumour development. These studies should also aim to identify the CUX1 isoform (or isoforms) that fulfils tumour-suppressing functions and confirm the mechanism (or mechanisms) involved: that is, transcriptional activation of PIK3IP1 by $\mathrm{p} 110$ CUX1 (REF. 11), DNA repair activities of p200 CUX1 (REF. 18), or both. If any tumours develop in a Cux $1^{+/-}$ mouse model, we should also investigate whether the remaining allele eventually becomes amplified during tumour progression. Indeed, although the frequency of CUX1 monoallelic inactivation and reduced expression is higher in certain cancer types, whereas increased copy number and expression occurs more often in other types of cancers, the two events can be found in cancers from the same tissues. Moreover, many tumour cell lines exhibit both CUX1 LOH and increased copy number of the remaining allele, suggesting that deletion of one allele and amplification of the other occur successively in the same tumour cells (FIG. 3). In addition, we should aim to identify changes in the circuitry of cancer cells that annihilate the tumour-suppressing function (or functions) of CUX1 while still permitting its stimulatory effects on proliferation, motility and resistance to apoptosis.

At the molecular level, it is likely that the role of p200 CUX1 in DNA repair is not limited to its stimulatory effect on OGG1 but involves additional interactions with other DNA repair proteins that could potentially be targeted in future therapeutic strategies. The synthetic lethality of CUX1 knockdown in RAS-driven cancer cells may have revealed the Achilles' heel of a type of cancer cells for which there is currently no targeted therapy ${ }^{18,83}$. Indeed, DNA repair mechanisms are implicated in cancer in multiple ways that may appear to be contradictory. Defects in DNA repair, whether transient or permanent, contribute to tumour development and progression. However, DNA repair pathways are also required for cancer cells to replicate their DNA and rapidly proliferate. Moreover, radiotherapy and most chemotherapeutic agents aim to kill cancer cells 
by causing DNA damage, and efficient DNA repair is now accepted to contribute to confer resistance to these agents $^{84,85}$. As previously stated by others, "we are now entering a new era of cancer research in which patients may be stratified for appropriate therapy on the basis of the DNA damage response status of their tumour, rather than on the tissue of origin" (REF. 84). It will be important to verify whether CUX1 expression and DNA repair activities have an impact on the resistance of cancer cells to treatments that cause DNA damage.
1. Blochlinger, K., Bodmer, R., Jack, J., Jan, L. Y. \& Jan, Y. N. Primary structure and expression of a product from cut, a locus involved in specifying sensory organ identity in Drosophila. Nature 333. 629-635 (1988).

2. Neufeld, E. J., Skalnik, D. G., Lievens, P. M. \& Orkin, S. H. Human CCAAT displacement protein is homologous to the Drosophila homeoprotein, cut. Nature Genet. 1, 50-55 (1992).

3. Schoenmakers, E. F. et al. Identification of $C U X 1$ as the recurrent chromosomal band $7 q 22$ target gene in human uterine leiomyoma. Genes Chromosomes Cancer 52, 11-23 (2013).

4. Jerez, A. et al. Loss of heterozygosity in 7q myeloid disorders: clinical associations and genomic pathogenesis. Blood 119, 6109-6117 (2012).

5. McNerney, M. E. et al. CUX1 is a haploinsufficient tumor suppressor gene on chromosome 7 frequently inactivated in acute myeloid leukemia. Blood 121 , 975-983 (2013).

6. Klampfl, T. et al. Genome integrity of myeloproliferative neoplasms in chronic phase and during disease progression. Blood 118, 167-176 (2011).

7. Hindersin, S., Niemeyer, C. M., Germing, U., Göbel, U. \& Kratz, C. P. Mutation analysis of CUTL1 in childhood myeloid neoplasias with monosomy 7. Leukemia Res. 31, 1323-1324 (2007).

8. Patrikis, M. et al. Mutation analysis of $C D P, T P 53$, and KRAS in uterine leiomyomas. Mol. Carcinogen. 37 61-64 (2003)

9. Moon, N. S. et al. Expression of N-terminally truncated isoforms of CDP/CUX is increased in human uterine leiomyomas. Int. J. Cancer 100, 429-432 (2002).

10. Thoennissen, N. H. et al. Prevalence and prognostic impact of allelic imbalances associated with leukemic transformation of Philadelphia chromosome-negative myeloproliferative neoplasms. Blood 115,

2882-2890 (2010).

References 3, 6 and 10 provide compelling evidence that CUX1 is the target of LOH at 7q22.1. Moreover, references 4 and 5 show that CUX1 mRNA and protein expression was reduced approximately twofold in leukaemic cells of affected patients.

11. Wong, C. C. et al. Inactivating CUX1 mutations promote tumorigenesis. Nature Genet. 46, 33-38 (2014).

This study shows that CUX1 harbours inactivating somatic mutations in a proportion of human cancers and provides functional evidence that inactivation of $c t$ in D. melanogaster or Cux 1 in mice promotes tumour development.

12. The Cancer Genome Atlas Network. Comprehensive molecular characterization of human colon and rectal cancer. Nature 487, 330-337 (2012).

13. Michl, P. et al. CUTL1 is a target of TGF $\beta$ signaling that enhances cancer cell motility and invasiveness. Cancer Cell 7, 521-532 (2005)

This study is the first to show that CUX1 mRNA and protein expression is increased by TGF $\beta$ and is required for TGF $\beta$-induced cell migration and invasion.

14. Ripka, S. et al. CUX1: target of Akt signalling and mediator of resistance to apoptosis in pancreatic cancer. Gut 59, 1101-1110 (2010)

This study shows that CUX 1 expression is stimulated downstream of the PI3K-AKT pathway and confers resistance to apoptosis.

15. Ledford, A. W. et al. Deregulated expression of the homeobox gene Cux-1 in transgenic mice results in downregulation of $\mathrm{p} 27^{\mathrm{kip} 1}$ expression during nephrogenesis, glomerular abnormalities, and multiorgan hyperplasia. Dev. Biol. 245, 157-171 (2002).

16. Cadieux, C. et al. Transgenic mice expressing the p75 CCAAT-displacement protein/Cut homeobox isoform develop a myeloproliferative disease-like myeloid leukemia. Cancer Res. 66, 9492-9501 (2006).
17. Cadieux, C. et al. Mouse mammary tumor virus $p 75$ and p110 CUX1 transgenic mice develop mammary tumors of various histologic types. Cancer Res. 69 7188-7197 (2009).

This study shows that increased expression of either p75 CUX 1 or $\mathrm{p} 110$ CUX 1 in mammary epithelial cells causes mammary tumours of various histological types.

18. Ramdzan, Z. M et al. RAS transformation requires CUX1-dependent repair of oxidative DNA damage. PLoS Biol. 12, e1001807 (2014).

This study shows that p200 CUX1 accelerates repai of oxidative DNA damage, prevents RAS-induced senescence and allows the emergence of tumours with spontaneous activating mutations in Kras. CUX1 knockdown is shown to be synthetic lethal in KRAS- and HRAS-driven human cancer cells.

19. Sansregret, L. et al. Cut homeobox 1 causes chromosomal instability by promoting bipolar division after cytokinesis failure. Proc. Natl Acad. Sci. USA 108, 1949-1954 (2011)

This study shows that $\mathrm{p} 110 \mathrm{CUX} 1$ activates expression of genes involved in the spindle assembly checkpoint and promotes bipolar mitosis in cells with more than two centrosomes. However, frequent merotelic chromosome attachments lead to aneuploidy and rapidly generate populations of genetic variants from which tumour cells emerge.

20. Siam, R. et al. Transcriptional activation of the Lats 1 tumor suppressor gene in tumors of CUX1 transgenic mice. Mol. Cancer 8, 60-70 (2009).

21. Sansregret, L. et al. The $\mathrm{p} 110$ isoform of the CDP/Cux transcription factor accelerates entry into $\mathrm{S}$ phase. Mol. Cell. Biol. 26, 2441-2455 (2006).

22. Truscott, M. et al. Carboxyl-terminal proteolytic processing of CUX1 by a caspase enables transcriptional activation in proliferating cells. J. Biol. Chem. 282, 30216-30226 (2007).

23. Vadnais, C. et al. CUX1 transcription factor is required for optimal ATM/ATR-mediated responses to DNA damage. Nucleic Acids Res. 40, 4483-4495 (2012)

24. Goulet, B. et al. Characterization of a tissue-specific CDP/Cux isoform, 75, activated in breast tumor cells. Cancer Res. 62, 6625-6633 (2002).

25. Moon, N. S. et al. S Phase-specific proteolytic cleavage is required to activate stable DNA binding by the CDP/Cut homeodomain protein. Mol. Cell. Biol. 21, 6332-6345 (2001)

26. Vanden Heuvel, G. B., Quaggin, S. E. \& Igarashi, P. A Unique variant of a homeobox gene related to Drosophila Cut is expressed in mouse testis. Biol. Reprod. 55, 731-739 (1996).

27. Maitra, U., Seo, J., Lozano, M. M. \& Dudley, J. P. Differentiation-induced cleavage of Cutl1/CDP generates a novel dominant-negative isoform that regulates mammary gene expression. $\mathrm{Mol}$. Cell. Biol. 26, 7466-7478 (2006)

28. Sansregret, L. $\&$ Nepveu, A. The multiple roles of CUX1: Insights from mouse models and cell-based assays. Gene 412, 84-94 (2008).

29. Skalnik, D. G., Strauss, E. C. \& Orkin, S. H. CCAAT displacement protein as a repressor of the myelomonocytic-specific gp91-phox gene promoter. J. Biol. Chem. 266, 16736-16744 (1991).

30. Lievens, P. M. J., Donady, J. J., Tufarelli, C. \& Neufeld, E. J. Repressor activity of CCAAT displacement protein in HL-60 myeloid leukemia cells. J. Biol. Chem. 270, 12745-12750 (1995).

31. Superti-Furga, G., Barberis, A., Schreiber, E. $\delta$ Busslinger, $\mathrm{M}$. The protein CDP, but not $\mathrm{CP} 1$, Footprints on the CCAAT region of the g-globulin gene in unfractionated B-cell extracts. Biochim. Biophys. Acta 1007, 237-242 (1989)

32. Stunkel, W., Huang, Z., Tan, S. H., O'Connor, M. J. \& Bernard, H. U. Nuclear matrix attachment regions of human papillomavirus type 16 repress or activate the E6 promoter, depending on the physical state of the viral DNA. J. Virol. 74, 2489-2501 (2000).
33. Pattison, S., Skalnik, D. G. \& Roman, A. Ccaat Displacement protein, a regulator of differentiationspecific gene expression, binds a negative regulatory element within the 5 ' end of the human papillomavirus type 6 long control region. J. Virol. 71, 2013-2022 (1997).

34. Cubelos, B. et al. Cux 1 and Cux2 regulate dendritic branching, spine morphology, and synapses of the upper layer neurons of the cortex. Neuron 66 , 523-535 (2010).

35. Ellis, T. et al. The transcriptional repressor CDP (Cutl1) is essential for epithelial cell differentiation of the lung and the hair follicle. Genes Dev. 15, 2307-2319 (2001).

36. Moon, N. S., Berube, G. \& Nepveu, A. CCAAT displacement activity involves Cut repeats 1 and 2, not the Cut homeodomain. J. Biol. Chem. 275, 31325-31334 (2000).

37. Mailly, F. et al. The human cut homeodomain protein can repress gene expression by two distinct mechanisms: active repression and competition for binding site occupancy. Mol. Cell. Biol. 16 5346-5357 (1996).

38. Dianov, G. L. \& Hubscher, U. Mammalian base excision repair: the forgotten archangel. Nucleic Acids Res. 41, 3483-3490 (2013).

39. Vadnais, C. et al. Long-range transcriptional regulation by the $\mathrm{p} 110 \mathrm{CUX} 1$ homeodomain protein on the ENCODE array. BMC Genomics 14, 258 (2013).

40. Goulet, B. et al. A cathepsin L isoform that is devoid of a signal peptide localizes to the nucleus in S Phase and processes the CDP/Cux transcription factor. Mol. Cell 14, 207-219 (2004).

41. Harada, R. et al. Genome-wide location analysis and expression studies reveal a role for $\mathrm{p} 110$ CUX 1 in the activation of DNA replication genes. Nucleic Acids Res. 36, 189-202 (2008)

42. Kedinger, V. et al. p110 CUX1 homeodomain protein stimulates cell migration and invasion in part through a regulatory cascade culminating in the repression of E-cadherin and occludin. J. Biol. Chem. 284 , 27701-27711 (2009).

43. Kuhnemuth, B. et al. CUX1 modulates polarization of tumor-associated macrophages by antagonizing NF-кB signaling. Oncogene http://dx.doi.org/10.1038/ onc. 2013.530 (2013)

This study presents results showing that TGF $\beta$ stimulates the expression of CUX1 in tumour-associated macrophages. In turn, CUX1 regulates cytokine expression to modulate the tumour microenvironment.

44. Ozisik, Y. Y., Meloni, A. M., Surti, U. \& Sandberg, A. A Deletion 7q22 in uterine leiomyoma. A cytogenetic review. Cancer Genet. Cytogenet. 71, 1-6 (1993).

45. Zeng, W. R. et al. Loss of heterozygosity and reduced expression of the CUTL1 gene in uterine leiomyomas. Oncogene 14, 2355-2365 (1997).

46. Zeng, W. R. et al. Refined mapping of the region of loss of heterozygosity on the long arm of chromosome 7 in human breast cancer defines the location of a second tumor suppressor gene at $7 q 22$ in the region of the CUTL1 gene. Oncogene 18, 2015-2021 (1999).

47. Pedersen-Bjergaard, J., Andersen, M. T. \& Andersen, M. K. Genetic pathways in the pathogenesis of therapy-related myelodysplasia and acute myeloid leukemia. Hematology Am. Soc. Hematol. Educ Program 392-397 (2007)

48. Zhang, Y. \& Rowley, J. D. Chromatin structural elements and chromosomal translocations in leukemia. DNA Repair 5, 1282-1297 (2006).

49. Thoennissen, N. H. et al. Novel CUX 1 missense mutation in association with 7q- at leukemic transformation of MPN. Am. J. Hematol. 86 703-705 (2011)

50. Luong, M. X. et al. Genetic ablation of the CDP/Cux protein $\mathrm{C}$ terminus results in hair cycle defects and reduced male fertility. Mol. Cell. Biol. 22, 1424-1437 (2002).

51. Sinclair, A. M. et al. Lymphoid apoptosis and myeloid hyperplasia in CCAAT displacement protein mutant mice. Blood 98, 3658-3667 (2001). 
52. Adzhubei, I. A et al. A method and server for predicting damaging missense mutations. Nature Methods 7, 248-249 (2010).

53. Kumar, P., Henikoff, S. \& Ng, P. C. Predicting the effects of coding non-synonymous variants on protein function using the SIFT algorithm. Nature Protoc. 4 1073-1081 (2009).

54. Collier, L. S., Carlson, C. M., Ravimohan, S., Dupuy, A. J. $\&$ Largaespada, D. A. Cancer gene discovery in solid tumours using transposon-based somatic mutagenesis in the mouse. Nature 436, 272-276 (2005).

55. Glosli, H. et al. Effects of hTNF $\alpha$ expression in T cells on haematopoiesis in transgenic mice. Eur. J. Haematol. 63, 50-60 (1999).

56. Lievens, P. M., Tufarelli, C., Donady, J. J., Stagg, A. \& Neufeld, E. J. CASP, a novel, highly conserved alternative-splicing product of the CDP/cut/cux gene, lacks cut-repeat and homeo DNA-binding domains, and interacts with full-length CDP in vitro. Gene 197 73-81 (1997)

57. Gillingham, A. K., Pfeifer, A. C. \& Munro, S. CASP, the alternatively spliced product of the gene encoding the CCAAT-displacement protein transcription factor, is a Golgi membrane protein related to giantin. $\mathrm{Mol}$. Biol. Cell 13, 3761-3774 (2002).

58. Ripka, S. et al. WNT5A - target of CUTL1 and potent modulator of tumor cell migration and invasion in pancreatic cancer. Carcinogenesis 28, 1178-1187 (2007).

59. Brantley, J. G., Sharma, M., Alcalay, N. I. Heuvel, G. B. V. Cux-1 transgenic mice develop glomerulosclerosis and interstitial fibrosis. Kidney Int. 63, 1240-1248 (2003).

60. Vanden Heuvel, G. B. et al. Hepatomegaly in transgenic mice expressing the homeobox gene Cux-1. Mol. Carcinog. 43, 18-30 (2005).

61. Cadieux, C. et al. Polycystic kidneys caused by sustained expression of Cux 1 isoform p75. J. Biol. Chem. 283, 13817-13824 (2008)

62. Truscott, M. Harada, R Vadnais, C., Robert, F. \& Nepveu, A. p110 CUX1 cooperates with E2F transcription factors in the transcriptional activation of cell cycle-regulated genes. Mol. Cell. Biol. 28 3127-3138 (2008).

63. Seguin, L. et al. CUX1 and E2F1 regulate coordinated expression of the mitotic complex genes Ect2, MgcRacGAP, and MKLP1 in S phase. Mol. Cell. Biol. 29, 570-581 (2009)

64. Aleksic, T. et al. CUTL1 promotes tumor cell migration by decreasing proteasome-mediated Src degradation. Oncogene 26, 5939-5949 (2007).
65. Kedinger, $\mathrm{V}, \&$ Nepveu, A. The roles of CUX1 homeodomain proteins in the establishment of a transcriptional program required for cell migration and invasion. Cell Adh. Migr. 4, 348-352 (2010)

66. Michl, P. \& Downward, J. CUTL1: a key mediator of TGF $\beta$-induced tumor invasion. Cell Cycle 5, 132-134 (2006)

67. Hulea, L. \& Nepveu, A. CUX1 transcription factors: from biochemical activities and cell-based assays to mouse models and human diseases. Gene 497. 18-26 (2012).

68. van Wijnen, A. J., Wright, K. L., Lian, J. B., Stein, J. L. \& Stein, G. S. Human H4 histone gene transcription requires the proliferation-specific nuclear factor HiNF-D. Auxiliary roles for HiNF-C (Sp1-like) and HiNF-A (high mobility group-like). J. Biol. Chem. 264 15034-15042 (1989).

69. Holthuis, J. et al. Tumor cells exhibit deregulation of the cell cycle histone gene promoter factor HiNF-D. Science 247, 1454-1457 (1990).

70. van Wijnen, A. J. et al. CDP/cut is the DNA-binding subunit of histone gene transcription factor HiNF-D: a mechanism for gene regulation at the G1/S phase cell cycle transition point independent of transcription factor E2F. Proc. Natl Acad. Sci. USA 93 11516-11521 (1996).

71. Truscott, M. et al. CDP/Cux stimulates transcription from the DNA polymerase $\alpha$ gene promoter. Mol. Cell. Biol. 23, 3013-3028 (2003).

72. Coqueret, O., Berube, G. \& Nepveu, A. The mammalian Cut homeodomain protein functions as a cell-cycle-dependent transcriptional repressor which downmodulates $\mathrm{p} 21 \mathrm{WAF} 1 / \mathrm{CIP} 1 / \mathrm{SDI} 1$ in S phase. EMBO J. 17, 4680-4694 (1998)

73. Sharma, M., Fopma, A., Brantley, J. G. \& Vanden Heuvel, G. B. Coexpression of Cux-1 and Notch signaling pathway components during kidney development. Dev. Dyn. 231, 828-838 (2004)

74. Sharma, M. et al. Differential expression of Cux-1 and p21 in polycystic kidneys from Pkd1 null and cpk mice. Kidney Int. 67, 432-442 (2005).

75. Sharma, M. et al. The homeodomain protein Cux 1 interacts with Grg4 to repress p27 kip 1 expression during kidney development. Gene 439, 87-94 (2009).

76. He, X. et al. PIK3IP1, a negative regulator of PI3K suppresses the development of hepatocellular carcinoma. Cancer Res. 68, 5591-5598 (2008).

77. Luo, J., Solimini, N. L. \& Elledge, S. J. Principles of cancer therapy: oncogene and non-oncogene addiction. Cell 136, 823-837 (2009).
78. Silkworth, W. T., Nardi, I. K., Scholl, L. M. \& Cimini, D. Multipolar spindle pole coalescence is a major source of kinetochore mis-attachment and chromosome missegregation in cancer cells. PLoS ONE 4, e6564 (2009).

79. Ganem, N. J., Godinho, S. A. \& Pellman, D. A mechanism linking extra centrosomes to chromosomal instability. Nature 460, 278-282 (2009).

80. Nigg, E. A. Centrosome aberrations: cause or consequence of cancer progression? Nature Rev. Cancer 2, 815-825 (2002).

81. Storchova, Z. \& Kuffer, C. The consequences of tetraploidy and aneuploidy. J. Cell Sci. 121, 3859-3866 (2008).

82. Gerlinger, M. et al. Intratumor heterogeneity and branched evolution revealed by multiregion sequencing. New Engl. J. Med. 366, 883-892 (2012).

83. Luo, J. et al. A genome-wide RNAi screen identifies multiple synthetic lethal interactions with the Ras oncogene. Cell 137, 835-848 (2009).

84. Curtin, N. J. DNA repair dysregulation from cancer driver to therapeutic target. Nature Rev. Cancer 12 801-817 (2012)

85. Shaheen, M., Allen, C., Nickoloff, J. A. \& Hromas, R. Synthetic lethality: exploiting the addiction of cancer to DNA repair. Blood 117, 6074-6082 (2011).

\section{Acknowledgements}

This work was supported by Grant number MOP-98010 from the Canadian Institutes of Health Research (CIHR) to A.N. Z.M.R. was supported by the Fonds de la recherche du Québec-Santé (FROS). This Review is dedicated to the memory of Rosalind Goodman.

\section{Competing interests statement}

The authors declare no competing interests.

\section{FURTHER INFORMATION}

Broad Institute Tumourscape website: http://www.

broadinstitute.org/tumorscape/pages/portalHome.jsf

Catalogue of Somatic Mutations in Cancer (COSMIC):

http://cancer.sanger.ac.uk/cosmic/gene/analysis? ln=CUX

PhosphoSitePlus database:

http://www.phosphosite.org/homeAction.do

Sanger cancer cell line website: http://cancer.sanger.ac.uk/

cancergenome/projects/cell_lines/

ALL LINKS ARE ACTIVE IN THE ONLINE PDF 